\title{
AVALIAÇÃO DA QUALIDADE DE SEMENTES DE CEDRO (Cedrela fissilis - Meliaceae) PELO TESTE DE RAIOS X
}

\author{
Evaluation of the seed quality in Cedrela fissilis - (Meliaceae) by X-ray test
}

\author{
Tathiana Elisa Masettoํㅜ José Márcio Rocha Faria², Sue Ellen Ester Queiroz ${ }^{3}$
}

\begin{abstract}
RESUMO
$\mathrm{O}$ teste de raios $\mathrm{X}$ tem se demonstrado uma técnica muito eficiente no controle de qualidade de sementes de espécies florestais, caracterizando fases do desenvolvimento, danos causados por secagem, beneficiamento e predação, entre outros. Objetivouse com este trabalho avaliar a qualidade física de sementes de cedro (Cedrela fissilis) pelo teste de raios X. Para isso, após o beneficiamento, as sementes de cedro foram expostas às seguintes condições de intensidade de radiação e tempos (25 Kv durante 60/ 30 segundos e $30 \mathrm{Kv}$ durante 40 segundos) para determinar o padrão de raios X. De acordo com a anatomia visualizada pela imagem radiográfica, as sementes foram classificadas em Sementes Cheias, Sementes Com Danos, Sementes Mal Formadas e Sementes Vazias. Em seguida, foi realizado o teste de germinação em BOD, utilizando-se substrato de papel umedecido dentro de caixas para germinação, sob $25^{\circ} \mathrm{C}$ e luz branca constante. Ao final de 20 dias, foram registrados os números de plântulas normais, anormais e sementes mortas. Pelos resultados obtidos, a intensidade de radiação de $25 \mathrm{Kv}$ durante 60 segundos permitiu melhor visualização das características internas e os danos observados pela imagem radiográfica afetaram a germinação das sementes, reduzindo a qualidade do lote.
\end{abstract}

Termos para indexação: Qualidade física de sementes, germinação, danos em sementes.

\begin{abstract}
The X-ray has showed to be a very efficient technique in the quality control of forest tree seeds, characterizing development phases, damages caused by dehydration, improvement and infestation, among others. The aim of this work was to evaluate the physical quality of cedar seeds (Cedrela fissilis) by X-ray test. For this, the cedar seeds were exposed at the following conditions of radiation intensity and times ( $25 \mathrm{Kv}$ during $60 / 30$ seconds and $30 \mathrm{Kv}$ during 40 seconds) to determine the pattern of X-rays. According to the anatomy visualized by the radiographic image, seeds were classified in Full Seeds; Seeds With Damages, Deformed Seeds and Empty Seeds. Soon after, the seeds were submitted to the germination test, accomplished in BOD, using paper substratum damped in boxes for germination under $25^{\circ} \mathrm{C}$ and white light. After 20 days, the numbers of normal, abnormal seedlings and died seeds were registered. From the results obtained the intensity of radiation of $25 \mathrm{Kv}$ in the time of exposure of 60 seconds allowed clear visualization of the internal characteristics and damages observed. By the radiographic damage affected the seed germination reducing the plot quality.
\end{abstract}

Index terms: Seeds physical quality, germination, damages in seeds.

\section{(Recebido em 22 de novembro de 2006 e aprovado em 2 de maio de 2007)}

\section{INTRODUÇÃO}

Vários testes podem estimar a qualidade de sementes, incluindo o teste de germinação, crescimento de plântulas, envelhecimento acelerado, condutividade elétrica e teste de tetrazólio, entre outros. Porém, a avaliação precisa e a aplicação desses testes às sementes de espécies florestais ainda é um entrave por causa da grande variabilidade genética que estas espécies apresentam (BONNER, 1998). Além disso, muitos destes testes são destrutivos, ou demandam longo tempo para a obtenção de resultados. Conseqüentemente, é importante continuar o desenvolvimento de novas alternativas que podem auxiliar a precisão e eficiência dos testes aplicados à qualidade de sementes florestais.

$\mathrm{O}$ teste de raios $\mathrm{X}$ foi introduzido na Suécia, por Simack e Gustafsson, em 1953, com o objetivo de avaliar a qualidade de sementes de algumas coníferas. O método consiste em expor as sementes a uma fonte de baixa energia de raios X e um filme fotossensível. Ao atravessarem as sementes e atingirem o filme, os raios permitem a formação de uma imagem latente caracterizada por diferentes graus de sombras e luz. O princípio da técnica é definido pela absorção de raios $\mathrm{X}$ em diferentes quantidades pelos tecidos das sementes, em função de suas estruturas,

\footnotetext{
'Engenheira Agrônoma, Doutoranda em Engenharia Florestal - Departamento de Ciências Florestais/DCF - Universidade Federal de Lavras/UFLA - Cx P. 3037 - 37200-000 - Lavras, MG - tmasetto@gmail.com

Engenheiro Florestal, PhD., Professor I - Departamento de Ciências Florestais/DCF - Universidade Federal de Lavras/UFLA - Cx. P. 3037 - $37200-000$ Lavras, MG - jmfaria@ufla.br

${ }^{3}$ Engenheira Florestal, Mestranda em Engenharia Florestal - Departamento de Ciências Florestais/DCF - Universidade Federal de Lavras/UFLA - Cx. P. 3037 - 37200-000 - Lavras, MG - suesinha01@yahoo.com.br
} 
composição e densidade, além do tempo de exposição à radiação (ISTA, 2004).

Essa técnica vem sendo aprimorada e já foi comprovada a sua eficiência na identificação de inúmeras características invisíveis a olho nu das sementes de várias espécies. Por exemplo, pode ser rapidamente visualizado o nível de maturidade dos embriões de Pinus silvestris (SAHLEN et al., 1995) e de sementes dessa espécie não germinadas ao final do teste (SIMAK et al., 1989); embriões mutantes de Arabidopsis thalliana (BINO et al., 1993); sementes de milho danificadas por estresse térmico no campo (CARVALHO et al., 1999) e mecanicamente danificadas (CICERO \& BANZATTO JÚNIOR, 2003) e fração de sementes puras de aveia (CRAVIOTTO et al., 2002) entre outros.

O cedro (Cedrela fissilis Vell.) pertence à família Meliaceae, é uma espécie nativa que ocorre desde o Rio Grande do Sul até Minas Gerais, principalmente nas florestas semidecídua e pluvial atlântica. Sua madeira é largamente empregada no setor moveleiro, construção civil, naval e aeronáutica. A árvore de cedro também é recomendada para o paisagismo e plantios heterogêneos de áreas degradadas (LORENZI, 1992). As sementes de cedro são largamente utilizadas para a produção de mudas em viveiro ou para semeadura direta no campo.

Até o momento, não há na literatura informações a respeito da utilização do teste de raios $\mathrm{X}$ para avaliação da qualidade de sementes de cedro. Por esse método não ser destrutivo, é possível diagnosticar de forma rápida a viabilidade das sementes durante e após o desenvolvimento e assim, decidir sobre o melhor período de coleta e ainda predizer a qualidade das sementes, o que otimizaria o processo de produção e armazenamento de sementes de cedro em bancos de germoplasma.

Assim, o objetivo desse trabalho foi avaliar a qualidade de sementes de cedro por meio de testes de raio $\mathrm{X}$.

\section{MATERIALE MÉTODOS}

Foram coletados frutos maduros em início de abertura (Figura 1) com auxílio de podão e lona plástica de aproximadamente 20 matrizes na região de Lavras, localizada ao sul de Minas Gerais ( $\left.21^{\circ} 14^{\prime} \mathrm{S}, 45^{\circ} 00^{\prime} \mathrm{W}\right)$, no mês de julho. Após a coleta, os frutos foram levados ao galpão do Laboratório de Sementes Florestais do Departamento de Ciências Florestais da Universidade Federal de Lavras (UFLA), MG. O beneficiamento consistiu de abertura dos frutos maduros e remoção do prolongamento alado de acordo com as recomendações de Davide et al. (1995).

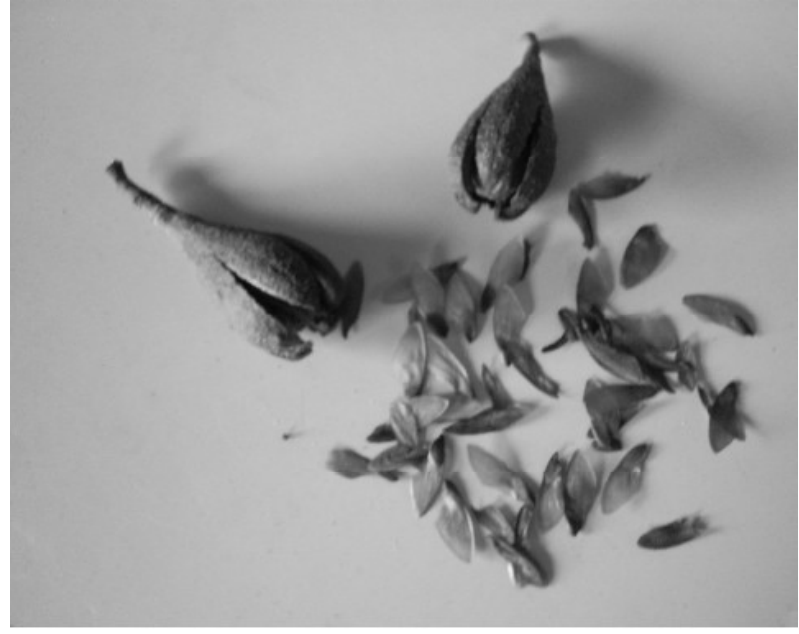

Figura 1 - Frutos e sementes de cedro (Cedrela fissillis). UFLA, Lavras, MG, 2006.

O grau de umidade foi determinado pelo método da estufa a $103 \pm 2^{\circ} \mathrm{C}$ por 17 horas (BRASIL, 1992), com 4 repetições de 2 gramas de sementes. Os resultados foram calculados com base no peso das sementes úmidas.

Inicialmente, foi realizado um teste de germinação com 4 repetições de 25 sementes que foram desinfetadas com hipoclorito de sódio $2 \%$ por 2 minutos e colocadas sobre papel umedecido dentro de caixas para germinação em BOD sob $25^{\circ} \mathrm{C}$ e luz branca constante. Para a realização do teste de raios $\mathrm{X}$, primeiramente foi determinada a melhor combinação de intensidade de radiação (Kvp) e tempo de exposição (Tabela 1). Utilizou-se o equipamento Faxitron HP, modelo 43855AX.

Tabela 1 - Intensidades de radiação e tempos de exposição das sementes de cedro (Cedrela fissilis) para o teste de raios X. UFLA, Lavras, MG, 2006.

\begin{tabular}{cc}
\hline $\begin{array}{c}\text { Intensidade de } \\
\text { radiação }(\mathrm{Kv})\end{array}$ & $\begin{array}{c}\text { Tempo de exposição } \\
\text { (segundos) }\end{array}$ \\
\hline 25 & 60 \\
25 & 30 \\
30 & 40 \\
\hline
\end{tabular}

Após a escolha da melhor intensidade e tempo de exposição, o teste de raios $\mathrm{X}$ foi realizado utilizando-se uma amostra aleatória de 400 sementes posicionadas sobre placa acrílica transparente $(2 \mathrm{~mm}$ de espessura e medindo $16 \times 17 \mathrm{~cm}$ ) e fixadas com fita dupla face. Em seguida, de 
acordo com a visualização da anatomia interna pelas radiografias, as sementes foram classificadas como Sementes Cheias (apresentavam um embrião bem formado), Mal Formadas (sementes que apresentavam um embrião, porém este ocupava apenas parte do tegumento), Com Danos (sementes que apresentavam lesão no embrião) e Vazias (aquelas que não apresentavam embrião). As sementes foram então identificadas, exceto as sementes Vazias, e submetidas ao teste de germinação da mesma forma citada anteriormente. $\mathrm{O}$ teste teve a duração de 20 dias e os números de sementes com plântulas normais, plântulas anormais e sementes mortas foram anotados ao final do teste.

Os dados obtidos no teste de germinação foram submetidos à análise de variância e às médias dos tratamentos comparadas pelo Teste de Tukey ao nível de $5 \%$ de probabilidade, usando o programa estatístico SISVAR.

\section{RESULTADOS E DISCUSSÃO}

Inicialmente, as sementes de cedro apresentaram $9 \%$ de teor de água após o beneficiamento e $70 \%$ de germinação. O baixo teor de água favorece a observação das características internas das sementes. De acordo com Simak (1991), quanto menor o conteúdo de água nas sementes, maior a densidade óptica, o que favorece a caracterização das estruturas internas.

A combinação de tempo de exposição de 60 segundos e intensidade de radiação de $25 \mathrm{Kv}$ permitiu a melhor visualização da anatomia interna das sementes de cedro. Os resultados obtidos pelo teste de germinação das sementes radiografadas constam na Tabela 2, e as imagens obtidas com a análise radiográfica das sementes constam na Figura 2. Pela análise radiográfica foi detectada a presença de $13 \%$ de sementes Vazias (Figura b), 35,25\% de Sementes Cheias (Figura d) que em sua maioria originaram plântulas normais (Figura e) ao final do teste de germinação, embora também apresentem sementes mortas. De acordo com Burg et al. (1994), devido à variação natural, algumas sementes, embora não apresentando danos pelo teste de raios $\mathrm{X}$, podem não germinar em conseqüência das alterações invisíveis por microrganismos ou danos fisiológicos.

As sementes classificadas como Mal Formadas (30\%) (Figura j) de acordo com a análise radiográfica, originaram plântulas anormais e em sua maioria sementes mortas. De acordo com os resultados, a deformação do embrião impossibilitou o desenvolvimento normal de plântulas (Figura k). A má formação de sementes florestais, como em aroeira (MACHADO \& CÍCERO, 2003) e canafístula (OLIVEIRA et al., 2003) visualizada por meio de teste de raios $\mathrm{X}$, resultou na morte de sementes nestas espécies. Para esses autores, o descarte dessas sementes pode melhorar a germinação do lote.

Por outro lado, foi observado que $21,75 \%$ das sementes pertenciam à classe de sementes Com Danos (Figura g), que em sua totalidade originaram plântulas anormais e sementes mortas ao final do teste (Figura $h$ ), indicando que os defeitos detectados na radiografia impediram a formação de plântulas normais ao final do teste de germinação e reduziram a qualidade do lote estudado.

Provavelmente, os danos detectados na análise radiográfica, localizados nos cotilédones, podem impedir a translocação de nutrientes para o eixo embrionário. Tais lesões podem ser originadas no momento do beneficiamento, o qual é realizado manualmente por meio de abertura dos frutos e separação das sementes. Independentemente da causa, tais lesões nos embriões não podem ser observadas a olho nu e prejudicam a qualidade do lote, como foi observado para as sementes de cedro.

Resultados semelhantes foram obtidos por Oliveira et al. (2004) para sementes de ipê- amarelo e ipê- roxo. Os defeitos internos, caracterizados por má formação e fissuras detectados pela radiografia, afetaram a germinação dessas sementes.

Pelos resultados da presente pesquisa, a aplicação do teste de raios $\mathrm{X}$ é especialmente promissora na análise de sementes de cedro, auxiliando a separação de lotes e a detecção de sementes vazias e com imperfeições.

A presença de insetos no interior das sementes de algumas espécies florestais, não presumíveis a olho nu, também pode ser facilmente visualizada por meio de análise radiográfica, como foi relatado para sementes de Eugenia pleurantha O. Berg. (MASETTO, 2005) e sementes do gênero Nectandra (CARVALHO, 2006).

Tabela 2 - Plântulas normais, anormais e sementes mortas de Cedrela fissilis encontradas para as categorias de Sementes Cheias, Mal Formadas e Sementes Com Danos pelo teste de raios X. Lavras, UFLA, MG, 2006.

\begin{tabular}{lccc}
\hline \multicolumn{1}{c}{ Categoria } & PN $(\%)$ & PA $(\%)$ & SM $(\%)$ \\
\hline Cheias & $96,66 \mathrm{a}$ & $0,00 \mathrm{a}$ & $3,34 \mathrm{a}$ \\
Com Danos & $63,33 \mathrm{~b}$ & $12,50 \mathrm{a}$ & $24,17 \mathrm{ab}$ \\
Mal Formadas & $0,00 \mathrm{c}$ & $50,00 \mathrm{~b}$ & $50,00 \mathrm{~b}$ \\
\hline
\end{tabular}

$\mathrm{CV}=15,23 \%$

Médias seguidas pela mesma letra na coluna não diferem entre si pelo Teste de Tukey, ao nível de 5\% de probabilidade. 

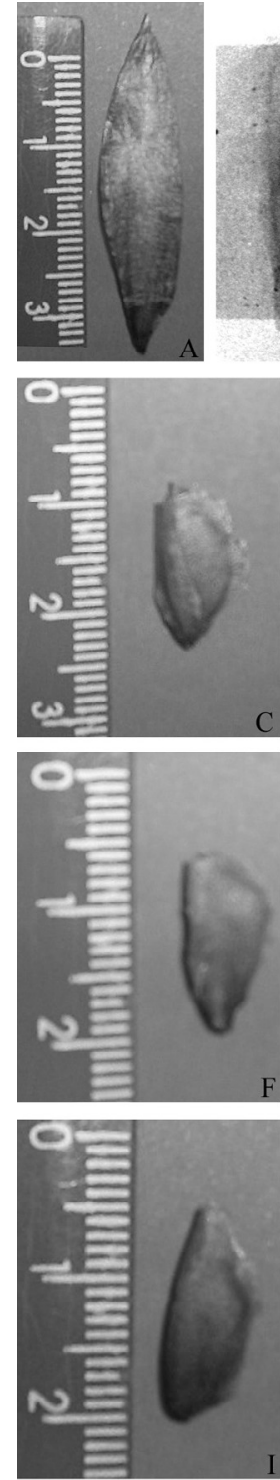

B
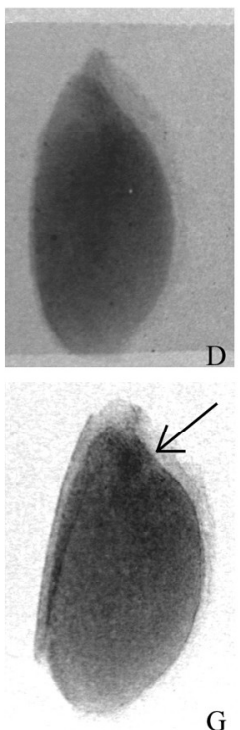

G
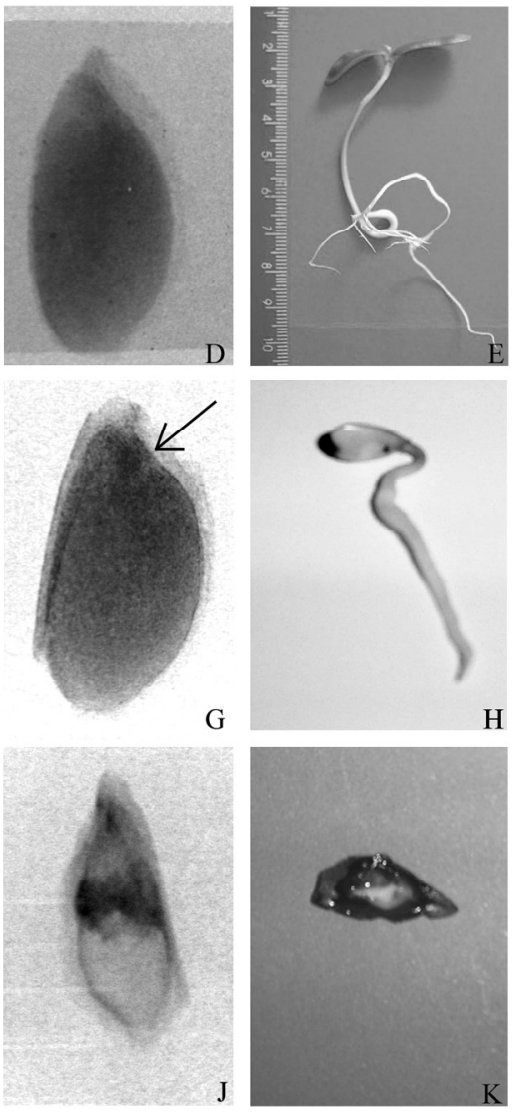

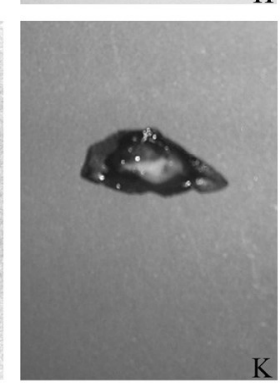

Figura 2 - Sementes de Cedrela fissilis visualmente intactas (a, c, f e i), classificadas pela análise radiográfica em Semente Vazia (b), Semente Cheia (d) Semente com Danos (g) e Semente Mal Formada (j); originando plântula normal (e), plântula anormal (h) e semente morta (k) ao final do teste de germinação. Lavras, MG, 2006.

$\mathrm{O}$ teste de raios $\mathrm{X}$ também pode ser utilizado para avaliação de danos causados por secagem em sementes (MASETTO et al., 2005). De acordo com Goodman et al. (2005), essa técnica foi eficiente para relacionar esses danos, detectados pela radiografia, com a perda da viabilidade e do vigor de sementes de Quercus rubra L. A condição morfológica da semente provavelmente reflete um resultado cumulativo de todos os fatores que influenciam a perda da viabilidade. Esses autores também afirmam que, com o contínuo desenvolvimento tecnológico da análise de imagem, essa técnica poderá ser aplicada para operações em larga escala e, também, em outros projetos de pesquisa em menor escala onde a avaliação da qualidade seja imediatamente requerida.

\section{CONCLUSÕES}

O teste de raios $\mathrm{X}$ é eficiente na detecção de danos em sementes de cedro. Os danos observados afetam negativamente a germinação das sementes, reduzindo a qualidade do lote.

\section{REFERÊNCIAS BIBLIOGRÁFICAS}

BINO, R. J.; AARTSE, J. W.; BURG, W. J. van der. Nondestructive X-ray of Arabidopsis embryo mutants. Seed Science Research, Wallingford, v. 3, n. 2, p. 167-170, June 1993.

BONNER, F. T. Testing tree seeds for vigor: a review. Seed Technology, Amsterdam, v. 20, p. 5-17, 1998.

BRASIL. Ministério da Agricultura. Regras para análise de sementes. Brasília, DF, 1992. 365 p.

BURG, W. J. van der; AARTSE, J. W.; ZWOL, R. A. van; JALINK, H.; BINO, R. J. Predicting tomato seedling morphology by X-ray analysis of seeds. Journal American Society for Horticultural Science, Alexandria, v. 119, n. 2, p. 258-263, Mar. 1994.

CARVALHO, L. R. Conservação de sementes do gênero Nectandra, Ocotea e Persea (Lauraceae). 2006. 60 p. Tese (Doutorado) - Universidade Federal de Lavras, Lavras, 2006.

CARVAlHO, M. L. M.; AESLT, A. C.; ECK, J. W.; HOEKSTRA, F. A. Pre-harvest stress cracks in maize (Zea mays L.) kernels as characterized by visual, X-ray and low temperature scanning electron microscopical analysis: effect on kernel quality. Seed Science Research, Wallingford, v. 9, p. 227-236, 1999.

CÍCERO, S. M.; BANZATTO JUNIOR, H. L. Avaliação do relacionamento entre danos mecânicos e vigor, em sementes de milho, por meio da análise de imagens. Revista Brasileira de Sementes, Brasília, v. 25, n. 1, p. 25-28, 2003. 
CRAVIOTTO, R. M.; YOLDJIAN, A. M.; SALINAS, A. R.; ARANGO, M. R.; BISARO, V.; MATURO, H. Description of pure seed fraction of oat through usual evaluations and radiographic images. Pesquisa Agropecuária Brasileira, Brasília, v. 37, n. 8, p. 1183-1188, Aug. 2002.

DAVIDE, A. C.; FARIA, J. M. R.; BOTELHO, S. A. Propagação de espécies florestais. Belo Horizonte: Cemig; Lavras: UFLA, 1995. 41 p.

GOODMAN, R. C.; JACOBS, D. F.; KARRFALT, R. P. Evaluating desiccation sensitivity of Quercus rubra acorns using X-ray image analysis. Canadian Journal Research, Ottawa, v. 35, p. 2823-2831, 2005.

INTERNATIONAL RULES FOR SEED TESTING. ISTA. Zurich, 2004. 180 p.

LORENZI, H. Árvores brasileiras: manual de identificação e cultivo de plantas arbóreas nativas do Brasil. Nova Odessa: Plantarum, 1992. 368 p.

MACHADO, C. F.; CICERO, S. M. Aroeira-branca (Lithraea molleoides (Vell.) Engl. - Anacardiaceae) seed quality evaluation by the X-ray test. Scientia Agricola, Piracicaba, v. 60, n. 2, p. 393-397, Apr./June 2003.

MASETTO, T. E. Estudo da sensibilidade à dessecação em sementes de Eugenia pleurantha (Myrtaceae). 2005. 60 p. Dissertação (Mestrado) - Universidade Federal de Lavras, Lavras, 2005.
MASETTO, T. E.; DAVIDE, A. C.; TONETTI, O. A. O. Utilização da técnica de raios $\mathrm{X}$ para avaliação do efeito de secagem em sementes de Eugenia handroana. In: CONGRESSO BRASILEIRO DE SEMENTES, 15., 2005, Foz do Iguaçu. Anais... Foz do Iguaçu: [s.n.], 2005.

OLIVEIRA, L. M.; CARVALHO, M. L. M.; DAVIDE, A. C. Utilização do teste de raios-X na avaliação da qualidade de sementes de canafístula (Peltophorum dubium (Sprengel) Taubert). Revista Brasileira de Sementes, Brasília, v. 25, n. 1, p. 116-120, 2003.

OLIVEIRA, L. M.; CARVALHO, M. L. M.; GUIMARÃES, R. M.; MASETTO, T. E. Avaliação da qualidade de sementes de Tabebuia Serratifolia Vahl Nich. E $T$. impetiginosa (Martius Ex A. P. De Candolle) Standley (BignONIACEAE) pelo teste de raios X. Revista Brasileira de Sementes, Brasília, v. 26, n. 2, p. 138-143, dez. 2004.

SAHLEN, K.; BERGSTEN, U.; WIKLUND, K. Determination of viable and dead Scots pine of different anatomical maturity after freezing using the IDX method. Seed Science Research, [S.1.], v. 23, p. 405-414, 1995.

SIMAK, M. Testing of forest tree and shrub seeds by Xradiography. In: GORDON, A. G.; GOSLING, P.; WANG, B. S. P. Tree and shrub seed handbook. Zurich: ISTA, 1991.

SIMAK, M.; BERGSTEN, U.; HENRIKSSON, G. Evaluation of ungerminated seeds at the end of germination test by radiography. Seed Science and Technology, Zurich, v. 17, p. $361-369,1989$. 